\title{
Three-dimensional characterisation of nanomaterials using aberration-corrected STEM
}

\author{
P.D. Nellist, E.C. Cosgriff, V.Nicolosi and J.N. Coleman
}

Trinity College Dublin, School of Physics, Dublin 2, IRELAND

The correction of spherical aberration in the scanning transmission electron microscope (STEM) has enabled dramatic improvements in spatial resolution, with corrected instruments now routinely achieving sub-angstrom resolution [1]. Here we explore the use of aberration-corrected STEM for determining the 3D structure of materials using two different approaches.

The improvement in resolution allows crystalline materials to be viewed from a greater number of crystallographic directions while still allowing the projected atomic spacings to be resolved. In the case of nanomaterials, the reduced number of atoms present suggests that only a few viewing directions are necessary to determine their structure. As an example of this, nanowire bundles of composition $\mathrm{Mo}_{6} \mathrm{~S}_{9-x} \mathrm{I}_{x}$ have diameters down to around $1 \mathrm{~nm}$ and are a class of material that are a promising alternative to carbon nanotubes [2]. Figure 1A shows how the nanowires kink and twist about their long axis, allowing images to be recorded from different crystallographic directions without tilting the sample. Figure $1 \mathrm{~B}$ shows a typical image where the wire is resolved with atomic resolution. The nanowire bundles consist of close packed arrays of individual one-dimensional molecular strands, so there are 3 high-symmetry directions along which the individual strands project cleanly. Previous X-ray diffraction and spectroscopic studies of this system have suggested space groups $\mathrm{P}_{3}(\# 173)$ or $\mathrm{R} \overline{3}$ (\#148) [3]. Both these symmetries suggest that all three high symmetry directions should give identical images, which is not observed in the STEM data. Further analysis of the STEM data allows a 3D structure for the nanowire to be determined that is in agreement with images from all three directions [manuscript in preparation].

An alternative approach to 3D characterisation makes use of the reduced depth of focus of an aberration-corrected STEM. Correction of spherical aberration increases the convergence angle leading to a dramatic reduction in depth of field which may be small as a few $\mathrm{nm}$ [4]. Given the ability of aberration corrected STEM to observe and obtain spectra from single dopant atoms within a more strongly diffracting crystalline matrix [5], it is now interesting to examine the extent to which optical sectioning through the reduction in the depth of focus can be used to determine such dopant depths. We have used a Bloch wave approach to compute the electron intensity in the illuminated As column of a sample of GaAs $<110>$ (Fig. 2). Underfocussing the lens to push the focal plane deeper into the crystal does indeed lead to a peak intensity occurring deeper in the crystal. However, the intensity maximum in general occurs before the expected plane, and can be up to $3.5 \mathrm{~nm}$ different. The Bloch wave approach will now allow us to analyse these results in terms of the channelling and non-channelling states [6].

[1] P.D. Nellist et al. Science 305 (2004) 1741.

[2] D. Vrbanic et al. Nanotechnology 15 (2004) 635.

[3] A. Meden et al. Nanotechnology 16 (2005) 1578.

[4] K. Van Benthem et al. Appl. Phys. Lett. 87 (2005) 034104.

[5] M. Varela et al. Phys. Rev. Lett. 92 (2004) 095502. 
[6] We would like to acknowledge the UK SuperSTEM facility for provision experimental facilities, and the Irish Higher Education Authority for financial support. We are extremely grateful to D. Vengust, D. Dvorsek and D. Mihailovic for supplying the nanowire material (see www.mo6.com for further details).

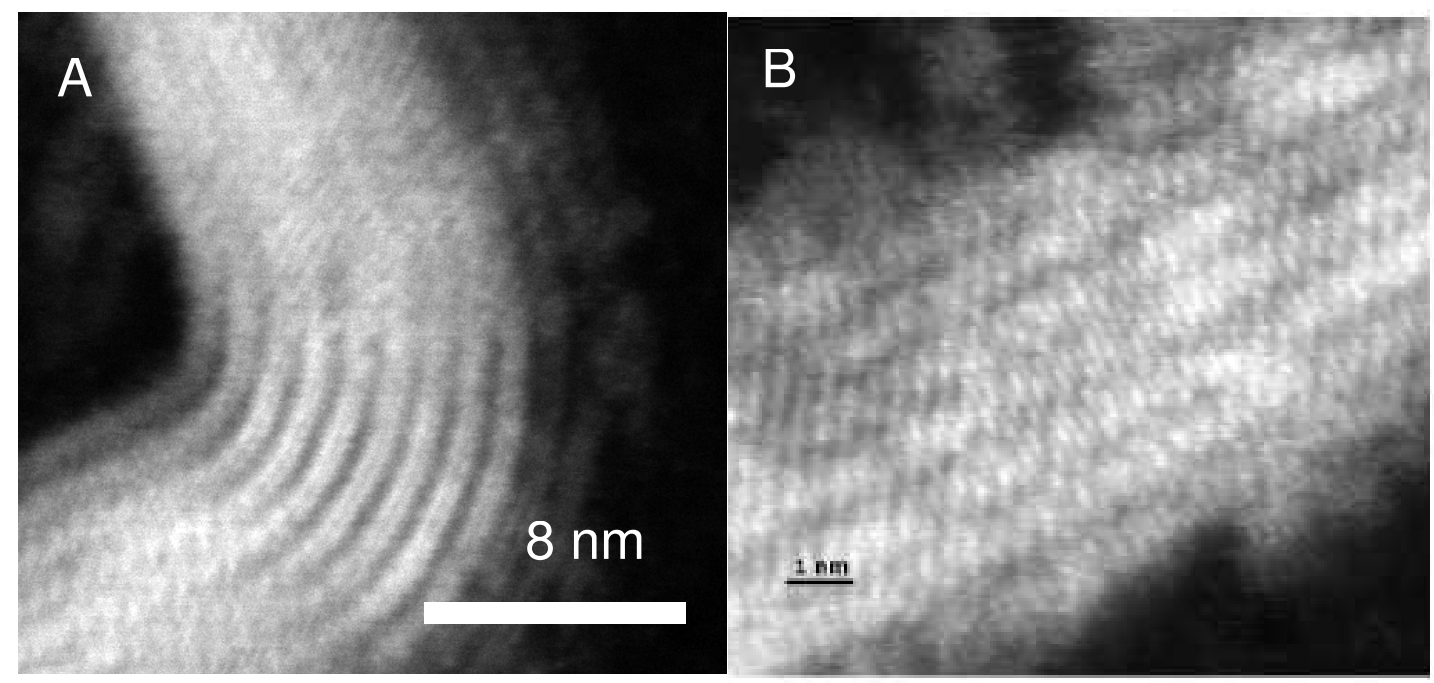

FIG. 1. (A) An annular dark-field (ADF) STEM image of a $\mathrm{Mo}_{6} \mathrm{~S}_{4.5} \mathrm{I}_{4.5}$ nanowire bundle showing a kink and the rotation of the bundle through a high symmetry direction. (B) An ADF STEM image of a $\mathrm{Mo}_{6} \mathrm{~S}_{3} \mathrm{I}_{6}$ nanowire bundle along a high symmetry direction showing the atomic structure resolved.
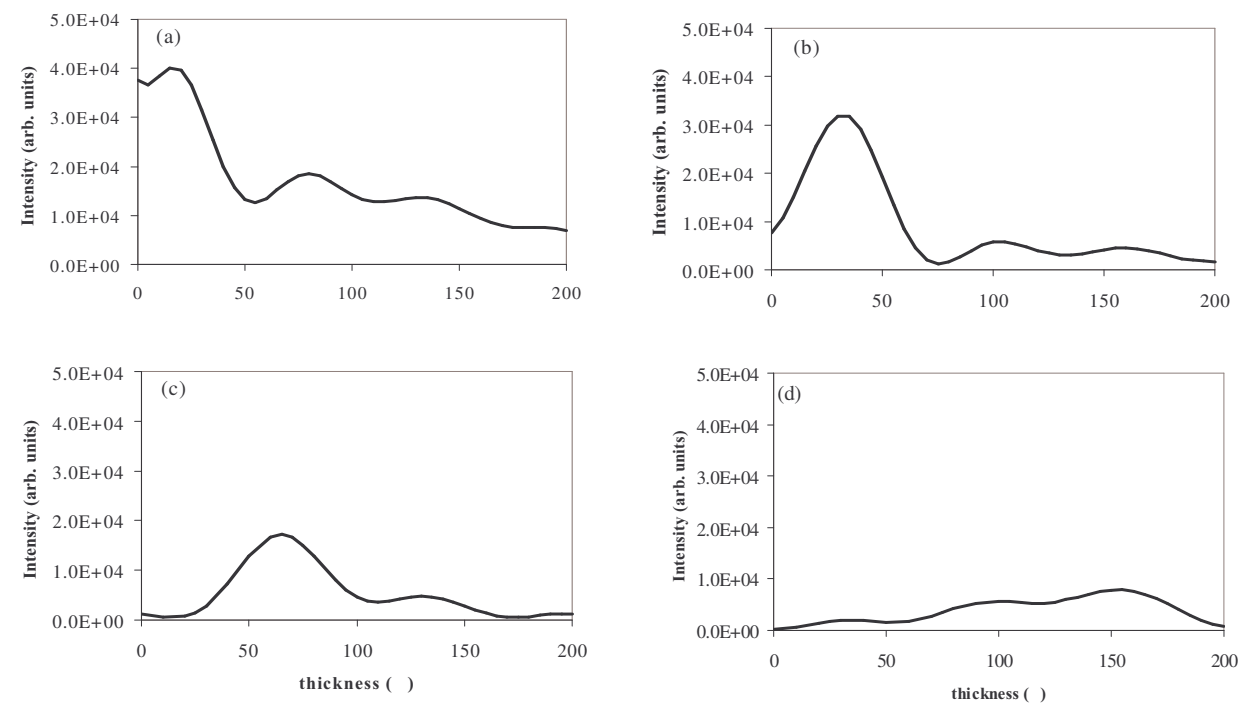

FIG. 2. The intensity within a $0.02 \mathrm{~nm}$ radius of the centre of the As column versus depth in the crystal for a sample of GaAs $<110>$ using $300 \mathrm{kV}$ electrons with a convergence angle of $22 \mathrm{mrad}$ and the probe located over the As column. The focal plane was set to the following depths in the crystal (a) 0 (entrance surface), (b) $5 \mathrm{~nm}$, (c) $10 \mathrm{~nm}$, (d) $15 \mathrm{~nm}$. 